\title{
Relation entre température et intensité des circulations d'eau souterraine dans les massifs alpins: outil de prévision des venues d'eau dans les tunnels \\ Relation between temperature and intensity of groundwater circulation in alpine massifs as a tool for predicting water inflows in tunnels
}

\section{J. C. Marechal}

Volume 12, numéro 3, 1999

URI : https://id.erudit.org/iderudit/705363ar

DOI : https://doi.org/10.7202/705363ar

Aller au sommaire du numéro

Éditeur(s)

Université du Québec - INRS-Eau, Terre et Environnement (INRS-ETE)

ISSN

0992-7158 (imprimé)

1718-8598 (numérique)

Découvrir la revue

Citer cet article

Marechal, J. C. (1999). Relation entre température et intensité des circulations d'eau souterraine dans les massifs alpins: outil de prévision des venues d'eau dans les tunnels. Revue des sciences de l'eau / Journal of Water Science, 12(3),

515-528. https://doi.org/10.7202/705363ar

\section{Résumé de l'article}

Un nombre important de grands tunnels profonds ont été, ou seront à l'avenir percés dans les massifs montagneux. Le suivi de l'évolution de la température des venues d'eau dans cinq grands tunnels alpins (Vereina, Gothard-N2, Mont-Blanc, Simplon et Gothard-AT) montre que la température de l'eau dans les ouvrages est fortement influencée par la perméabilité des massifs et les circulations d'eau souterraine qui s'y produisent. Les eaux froides qui s'infiltrent à haute altitude possèdent un effet réfrigérant sur le massif. Dès lors, la mesure de la température de l'eau en cours d'avancement d'un ouvrage souterrain constitue un outil de prévision efficace et peu coûteux des venues d'eau. Celles-ci peuvent être très localisées et provoquer une diminution de la température dans une zone du massif ou être diffuses et provoquer une diminution globale du gradient thermique des eaux dans le massif. Une corrélation négative a été mise en évidence entre le gradient thermique des eaux dans chacun des massifs étudiés et l'intensité des venues d'eau qui ont été ensuite observées dans les ouvrages les traversant. 


\title{
Relation entre température et intensité des circulations d'eau souterraine dans les massifs alpins : outil de prévision des venues d'eau dans les tunnels
}

\author{
Relation between temperature and intensity \\ of groundwater circulation in alpine massifs \\ as a tool for predicting water inflows in tunnels
}

\section{J.C. MARÉCHAL *}

Reçu le 5 novembre 1998, accepté le 21 janvier 1999**.

\section{SUMMARY}

Numerous long tunnels have been and will be drilled at great depths in mountainous alpine massifs. Water inflow temperatures in five existing long alpine tunnels (Vereina, Gothard-N2, Mont-Blanc, Simplon and Gothard-AT) have been studied and compared with the volume of water inflows.

The Vereina railway tunnel drilled in Austroalpine nappes encountered little water inflow. The linear discharge rates vary between 0.003 and $0.006 \mathrm{~L} / \mathrm{s} / \mathrm{m}$. Water temperatures series have been observed in both the northern and southern parts of the tunnel trace: the northern thermal gradient is equal to $0.018^{\circ} \mathrm{C} / \mathrm{m}$, whereas the southern thermal gradient is not very different with a value equal to $0.016^{\circ} \mathrm{C} / \mathrm{m}$. No special thermal anomaly has been observed at this site.

The Gothard-N2 road tunnel (National route number two) intersects the Aar and Gothard External Crystalline Massifs. A general thermal gradient equal to $0.015^{\circ} \mathrm{C} / \mathrm{m}$ is observed in the southern part of the tunnel trace in the Monte Prosa massif. Positive thermal anomalies have been measured in both the northern and central parts of the tunnel trace. They are due to topographical effects: in this region, the tunnel is situated beneath the Reuss river valley. Water inflows are weak in this tunnel: about $0.020 \mathrm{~L} / \mathrm{s} / \mathrm{m}$ in the Monte Prosa zone, for example.

The Mont-Blanc road tunnel intersects the Mont-Blanc External Crystalline Massif. A water thermal gradient equal to $0.016^{\circ} \mathrm{C} / \mathrm{m}$ has been observed on the northern part of the massif, at depths less than 1000 meters. This region corresponds to a low-permeability crystalline schist zone. The linear discharge rate is equal to $0.008 \mathrm{~L} / \mathrm{s} / \mathrm{m}$ in this zone. A large negative thermal

Laboratoires de Géologie de l'Ingénieur, Hydrogéologie et Prospection Géophysique, Université de Liège, Bâtiment B-19, 4000 Liège, Belgique.

* Correspondance. E-mail : jc.marechal@ulg.ac.be

* * Les commentaires seront reçus jusqu'au 10 mai 2000 
anomaly was measured during the drilling of this tunnel. The water temperatures decreased from $32^{\circ} \mathrm{C}$ to $11.5^{\circ} \mathrm{C}$ beneath the Pointe Helbronner. This decrease corresponds to large water inflows (about $1000 \mathrm{~L} / \mathrm{s}$ ) in a strongly fractured zone. A second water thermal gradient (very weak: $0.007^{\circ} \mathrm{C} / \mathrm{m}$ ) corresponds to the granitic unit which is globally more permeable than the schist with a linear discharge rate equal to $0.193 \mathrm{~L} / \mathrm{s} / \mathrm{m}$.

The Simplon railway tunnel, drilled through the Penninic nappes, is also characterized by a negative thermal anomaly situated in the very permeable marbles of the Teggiolo zone. In this tunnel, the water temperatures decrease from $55^{\circ} \mathrm{C}$ in the Berisal gneissic zone to less than $15^{\circ} \mathrm{C}$ in the Teggiolo zone. The water thermal gradient in the northern part is high, in conformity with the weak water inflows (linear discharge rate less than $0.001 \mathrm{~L} / \mathrm{s} / \mathrm{m}$ ). A third zone is observed in the Veglia marbles: it is characterized by a water thermal gradient equal to $0.010^{\circ} \mathrm{C} / \mathrm{m}$ for a linear discharge rate equal to $0.203 \mathrm{~L} / \mathrm{s} / \mathrm{m}$.

The Gothard-AT gallery has been drilled in Penninic gneiss. A water thermal gradient equal to $0.013^{\circ} \mathrm{C} / \mathrm{m}$ has been measured over the first $3000 \mathrm{~m}$. A negative thermal anomaly was encountered at the end of the gallery, due to the presence of very permeable metasedimentary rocks with important water circulation.

These results show that the water temperature in underground works is strongly dependent on the massif permeability and the existence of groundwater flows. Cold waters coming from high infiltration zones have a refrigerating effect on the massif. Thus, measuring water temperature during drilling constitutes a prediction tool for eventual water inflows. Two cases are possible: the observation of a local thermal anomaly due to a very localized aquifer zone, or the decrease of the water thermal gradient due to diffuse water inflows in the massif.

Local thermal anomalies, correlated with large water inflows along discrete zones, have been shown in the Simplon, Mont-Blanc and Gothard-AT tunnels. Such thermal anomalies can be measured hundreds of meters before the intersection of the tunnel with the aquifer zone: temperature monitoring thus constitutes a prediction tool for large water inflows localized in a particular aquifer zone. The use of $3 D$ numerical simulations allows one to improve the prediction quantitatively, by taking into account the problem geometry, the heterogeneity and anisotropy of the thermal and hydrogeological properties of rocks, and the boundary conditions.

The comparison of water thermal gradients at the massif scale with linear discharge rates in the tunnels through the massif allows us to determine a mathematical relationship between these characteristics of the massif. This relation permits one to predict the water quantity expected during drilling, knowing the water thermal gradient.

These results show that water temperature measurements during drilling of an underground work constitute an efficient and cheap predicting tool for water inflows. Anomalies due to relief must be taken into account; these can be very important in such mountainous massifs. A 3D modeling of heat transfer in the massif is, in all cases, necessary to improve the precision of predictions.

Key-words: temperature, Alps, undergrund work, hydrogeology, mountain.

\section{RÉSUMÉ}

Un nombre important de grands tunnels profonds ont été, ou seront à l'avenir, percés dans les massifs montagneux. Le suivi de l'évolution de la température des venues d'eau dans cinq grands tunnels alpins (Vereina, Gothard-N2, Mont-Blanc, Simplon et Gothard-AT) montre que la température de l'eau 
dans les ouvrages est fortement influencée par la perméabilité des massifs et les circulations d'eau souterraine qui s'y produisent. Les eaux froides qui s'infiltrent à haute altitude possèdent un effet réfrigérant sur le massif. Dès lors, la mesure de la température de l'eau en cours d'avancement d'un ouvrage souterrain constitue un outil de prévision efficace et peu coûteux des venues d'eau. Celles-ci peuvent être très localisées et provoquer une diminution de la température dans une zone du massif ou être diffuses et provoquer une diminution globale du gradient thermique des eaux dans le massif. Une corrélation négative a été mise en évidence entre le gradient thermique des eaux dans chacun des massifs étudiés et l'intensité des venues d'eau qui ont été ensuite observées dans les ouvrages les traversant.

Mots clés : température, Alpes, ouvrage souterrain, hydrogéologie, montagne.

\section{1 - INTRODUCTION}

Les différentes voies de communication nord - sud au travers des Alpes ont nécessité le creusement de plusieurs ouvrages souterrains à grande profondeur: des exemples sont les tunnels routiers de Tende, du Mont-Cenis, du MontBlanc, du Grand-Saint-Bernard ou du Gothard et les tunnels ferroviaires du Loetschberg, du Gothard ou du Simplon. Diverses galeries hydroélectriques de grands barrages se situent également dans les massifs alpins : les aménagements hydroélectriques EDF en France, les galeries de Grande Dixence dans le Valais suisse ou les galeries hydroélectriques dans le massif de l'Aar. A l'avenir, divers projets d'ouvrages souterrains concernent encore les régions alpines. Que ce soit pour des voies de communication (projets de ferroutage AlpTransit en Suisse, projets autoroutiers du Mercantour et de Tende dans le sud de la France et le nord de l'Italie, ou encore projets ferroviaires dans le nord de l'Italie avec les projets Mont-Cenis, Grand-Saint-Bernard, Simplon et Brenner à l'Est de l'Autriche) ou pour des aménagements hydrauliques (projets Cleuson-Dixence en Suisse et Nouvelle Romanche en France), de nombreux ouvrages souterrains vont encore être creusés dans les massifs montagneux alpins.

Dans le cadre d'un projet de grand tunnel sous les Alpes, il est essentiel de prévoir les conditions de travail des hommes et des machines qui vont réaliser l'ouvrage. Parmi tous les problèmes géotechniques qui doivent être résolus, la prévision des venues d'eau drainées et de leur température est une nécessité avant le choix des méthodes de perforation, de ventilation, de drainage et de refroidissement en cours de percement, mais aussi en cours d'exploitation de l'ouvrage.

En général, le gradient géothermique varie avec le flux géothermique et la conductivité des roches. En montagne, les choses sont compliquées par le relief : le gradient géothermique est plus fort au niveau des vallées que sous les sommets. Par ailleurs, les hydrogéologues et géophysiciens ont reconnu depuis longtemps la forte influence des circulations d'eau souterraine sur la distribution des températures en profondeur. Les premiers travaux entrepris dans le domaine ont montré cette dépendance (DONALDSON, 1962 ; STALLMAN, 1963) ; ensuite, les chercheurs ont utilisé celle-ci pour déterminer les propriétés hydrauliques d'un aquifère (BAIR et PARIZEK, 1978), pour prospecter des aqui- 
fères peu profonds (CARTWRIGHT, 1968) ou pour déterminer des zones d'écoulement souterrain (KEYS et BROWN, 1978 ; TANIGUCHI, 1993). À une échelle plus régionale, les effets thermiques des écoulements souterrains ont été mis en évidence et étudiés grâce notamment aux modèles numériques (SMITH et CHAPMAN, 1983 ; WOODBURY et SMITH, 1985). Dans le cadre de la réalisation d'ouvrages souterrains en milieu montagneux, les études sont restées longtemps focalisées sur la prévision des températures dans l'ouvrage (RYBACH et PFISTER, 1994A ; 1994B ; GOY et al., 1996). Tout récemment, des recherches ont été entreprises pour prévoir grâce aux températures mesurées en cours de percement, les quantités d'eau drainées par l'ouvrage (BUSSLINGER et RYBACH, 1996 ; 1997 ; MARÉCHAL et al., 1999). C'est à ce dernier aspect du problème que s'attache le présent article.

Les eaux drainées par les tunnels en montagne sont généralement caractérisées par un accroissement de leur température de l'entrée de l'ouvrage à proximité des versants vers le cœur des massifs montagneux. Ce phénomène résulte de l'augmentation de l'épaisseur de couverture rocheuse au-dessus de l'ouvrage. II est dès lors possible, sur un diagramme températures - épaisseur de couverture, de déterminer le gradient thermique des eaux qui caractérise l'accroissement de température des eaux par mètre de couverture rocheuse audessus du tunnel. Ce gradient thermique est affecté non seulement par les caractéristiques géothermiques du site (propriétés thermiques des terrains, flux géothermique) mais également par la forme du relief et l'existence éventuelle de circulations d'eau.

La démarche adoptée dans ce travail consiste à suivre l'évolution de la température de l'eau en fonction de l'épaisseur de la couverture rocheuse dans cinq tunnels profonds réalisés dans les Alpes Nereina, Gothard-N2, MontBlanc, Simplon et Gothard-AT) et la comparer aux quantités d'eau drainées par l'ouvrage en cours de percement, afin de déterminer l'incidence des eaux et d'autres facteurs tel le relief sur le champ thermique.

Après le percement d'un tunnel, les températures observées évoluent généralement suite à la modification des systèmes d'écoulement d'eau souterraine et au rôle réfrigérant de l'ouvrage. Les températures prises en compte dans ce travail sont dès lors celles mesurées en cours de percement et correspondant à la situation initiale non modifiée par la présence de l'ouvrage.

\section{2 - OBSERVATIONS ET INTERPRÉTATIONS}

\section{Tunnel de la Vereina}

Le tunnel ferroviaire de la Vereina, long de 19048 mètres, a été percé en Suisse ces dernières années dans les gneiss et amphibolites de la nappe austroalpine de Silvretta, sous une couverture rocheuse maximale égale à 1750 mètres (KELLER et LOCHER, 1994). Dans cet ouvrage (figure 1), la température des eaux est comprise entre $9,2^{\circ} \mathrm{C}$ à 410 mètres et $23,4^{\circ} \mathrm{C}$ à 12920 mètres de l'entrée nord. Les tronçons nord et sud, sensiblement symétriques, sont caractérisés par des gradients thermiques des eaux très semblables $\left(G_{\text {nord }}\right.$ $=0,018^{\circ} \mathrm{C} / \mathrm{m}$ et $\left.\mathrm{G}_{\text {sud }}=0,016^{\circ} \mathrm{C} / \mathrm{m}\right)$. On ne constate pas d'anomalie thermique 

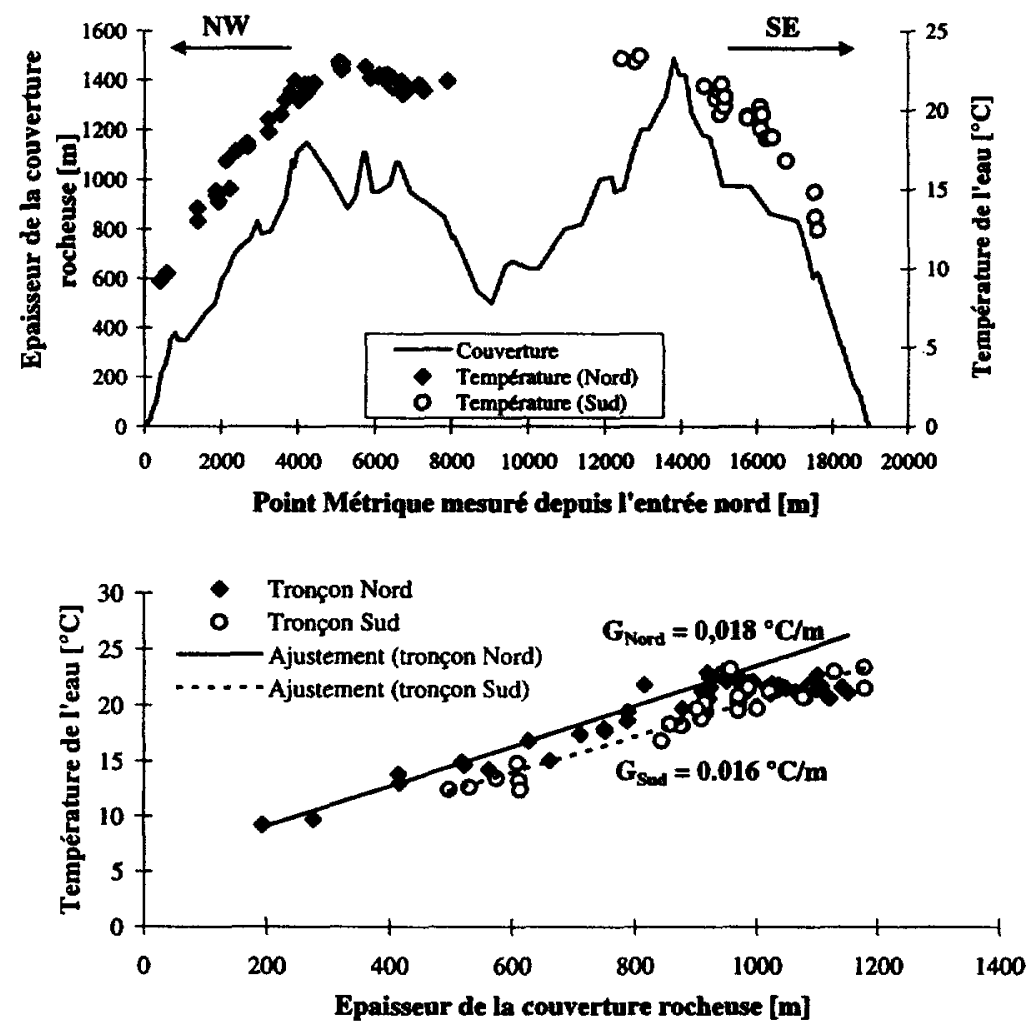

Figure 1 Cas de la Vereina.

(a) Profil de la température des venues d'eau et de l'épaisseur de la couverture rocheuse

(b). Évolution de la température de l'eau en fonction de l'épaisseur de la couverture rocheuse.

\section{Vereina case.}

(a) Profiles of water inflows temperatures and rock cover thickness.

(b) Water temperature as a function of rock cover thickness.

majeure dans cet ouvrage. Les venues d'eau sont peu importantes : les débits linéaires le long des deux tronçons sont faibles et comparables $(0,003 \mathrm{l} / \mathrm{s} / \mathrm{m}$ au nord et $0,006 \mathrm{l} / \mathrm{s} / \mathrm{m})$.

\section{Tunnel du Gothard-N2}

Le tunnel routier du Gothard fait partie de l'aménagement de la route nationale N2 en Suisse. Percé durant les années 70 , il traverse sur une longueur totale de 16322 mètres, les massifs cristallins externes du Gothard et de l'Aar sous une couverture rocheuse maximale de 1450 mètres (KELLER et al., 1987). Dans cet ouvrage, les températures des venues d'eau augmentent du sud vers le nord sous le massif du Monte Prosa (KELLER et al., 1987) : elles sont comprises entre $6^{\circ} \mathrm{C}$ à 170 mètres et $28,8^{\circ} \mathrm{C}$ à 4200 mètres de l'entrée sud sous le 
sommet du massif. Lorsque l'épaisseur de la couverture diminue vers le nord, les températures restent élevées dans la zone de Guspis où est d'ailleurs atteint le maximum mesuré : $32,4^{\circ} \mathrm{C}$ à 7000 mètres de l'entrée sud (figure 2a). Cette anomalie positive apparaît clairement sur le diagramme des épaisseurs de couverture - températures (figure $2 b$ ). Le tracé du tunnel a été choisi de manière à pouvoir implanter dans la zone de Guspis, un puits d'aération à moindre coût suite à la plus faible couverture. Ainsi, en surface, cette zone de Guspis est un point topographiquement bas situé au confluent de plusieurs vallées avec de tous côtés des sommets à plus de $2600 \mathrm{~m}$. L'anomalie apparente de température est donc d'origine topographique tout comme tout le tronçon nord du tunnel situé sous la vallée de la Reuss pour la même raison purement technique. Dans la zone du Monte Prosa, où le versant est régulier, l'accroissement de température est linéaire. II est dès lors possible de calculer le gradient thermique des eaux qui vaut $0,015^{\circ} \mathrm{C} / \mathrm{m}$ pour un faible débit linéaire égal à $0,020 \mathrm{~V} / \mathrm{s} / \mathrm{m}$.
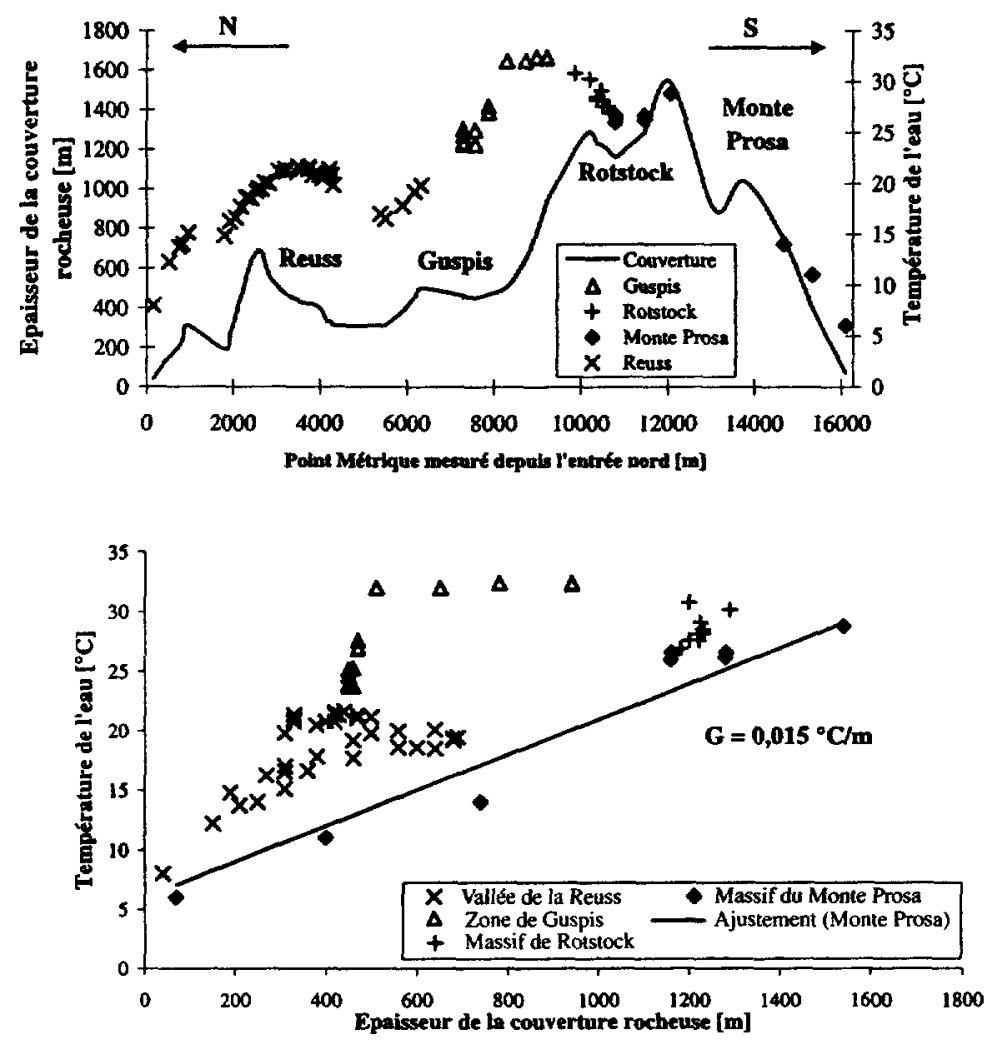

Figure 2 Cas du Gothard-N2.

(a) Profil de la température des venues d'eau et de l'épaisseur de la couverture rocheuse.

(b) Évolution de la température de l'eau en fonction de l'épaisseur de la couverture rocheuse.

Gothard-N2 case.

(a) Profiles of water inflows temperatures and rock cover thickness.

(b) Water temperature as a function of rock cover thickness. 

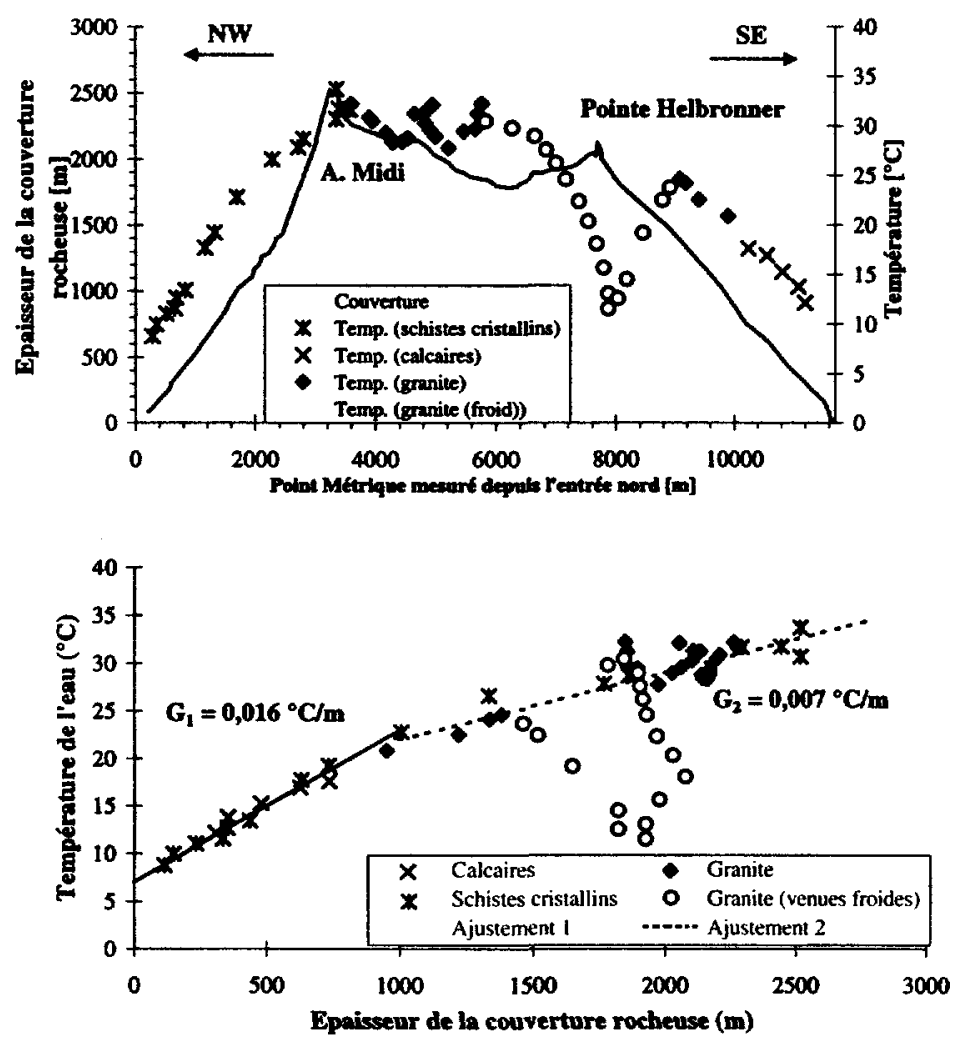

Figure 3 Cas du Mont-Blanc.

(a) Profil de la température des venues d'eau et de l'épaisseur de la couverture rocheuse.

(b) Évolution de la température de l'eau en fonction de l'épaisseur de la couverture rocheuse.

Mont-Blanc case.

(a) Profiles of water inflows temperatures and rock cover thickness.

(b) Water temperature as a function of rock cover thickness.

\section{Tunnel du Mont-Blanc}

Le tunnel routier du Mont-Blanc, percé au début des années 60 relie les vallées d'Aoste (l) et Chamonix (F). Long de 11600 mètres, il traverse le massif cristallin du Mont-Blanc constitué principalement de schistes cristallins et de granite, sous une épaisse couverture rocheuse (2 600 mètres au maximum). Dans cet ouvrage, les températures des venues d'eau (GUDEFIN, 1967) sont comprises entre $8,5^{\circ} \mathrm{C}$ à 280 mètres et $33,5^{\circ} \mathrm{C}$ à 3350 mètres de l'entrée nord. Une anomalie négative (température minimale égale à $11,5^{\circ} \mathrm{C}$ ) est présente dans une zone à fortes venues d'eau (à peu près $1000 \mathrm{l} / \mathrm{s}$ ) sous la Pointe Helbronner (figure 3a). Sur le diagramme des températures mesurées à l'avancement en fonction de l'épaisseur de la couverture (figure $3 b$ ), cette anomalie 
apparaît clairement. Deux gradients thermiques des eaux différents peuvent être déterminés. Un premier gradient $\left(G_{1}=0,016^{\circ} \mathrm{C} / \mathrm{m}\right)$ caractérise les venues d'eau pour lesquelles la couverture est inférieure à $1000 \mathrm{~m}$. II s'agit des venues d'eau rencontrées dans le tunnel à proximité du versant nord, dans les schistes cristallins. Le second gradient thermique $\left(G_{2}=0,007^{\circ} \mathrm{C} / \mathrm{m}\right)$, moins élevé, caractérise les venues d'eau situées au cour du massif sous plus de $1000 \mathrm{~m}$ de couverture rocheuse, dans le granite. Une modélisation mathématique des écoulements souterrains et des échanges de chaleur dans le massif (MARÉCHAL, 1998) a montré que c'est la présence de la zone fortement fracturée à grosses venues d'eau déjà à l'origine de l'anomalie de température négative et non la tendance habituelle en montagne d'un gradient plus élevé sous les vallées que sous les sommets, qui justifie cet écart de gradient. Le débit linéaire vaut en effet seulement $0,008 \mathrm{l} / \mathrm{s} / \mathrm{m}$ dans la zone des schistes cristallins à fort gradient thermique et augmente jusqu'à $0,193 \mathrm{l} / \mathrm{s} / \mathrm{m}$ dans la zone granitique à faible gradient thermique.

La chute de température, mesurée durant l'avancement plus de 1000 mètres avant la rencontre de la zone fortement aquifère, avait indiqué aux foreurs italiens l'imminence d'importantes venues d'eau (GUICHONET et BERNIERI, 1966). Une modélisation mathématique des écoulements souterrains et de la chaleur dans le massif du Mont-Blanc a permis de reconstituer cette anomalie thermique et de montrer qu'il est possible, en cours d'avancement, à partir de données de température, d'améliorer quantitativement la prévision des venues d'eau dans un ouvrage souterrain (MARÉCHAL et al., 1999).

\section{Tunnel du Simplon}

Une anomalie semblable à celle du Mont-Blanc a été mise en évidence dans le tunnel ferroviaire du Simplon où les variations de températures, suite aux contrastes de perméabilité des différentes unités penniques du massif, sont importantes. Percé au début du siècle entre la Suisse et l'Italie sous une épaisse couverture rocheuse (plus de 2000 mètres), cet ouvrage traverse les terrains cristallins du Pennique inférieur. Les températures atteignent $55^{\circ} \mathrm{C}$ (SCHARDT, 1905) au cœur du massif, à 8000 mètres de l'entrée nord, dans la zone à faible perméabilité de Berisal (gneiss schisteux) et baissent jusqu'à moins de $15^{\circ} \mathrm{C}$ dans les formations de marbres et calcschistes de Teggiolo, karstifiées et siège d'importantes circulations d'eau, à 15600 mètres de l'entrée nord (figure 4a). Le gradient thermique des eaux $\left(\mathrm{G}_{1}=0,023^{\circ} \mathrm{C} / \mathrm{m}\right)$ est élevé dans la partie sud, suite à l'absence d'eau souterraine (débit linéaire inférieur à $0,001 \mathrm{l} / \mathrm{s} / \mathrm{m})$. Plus au nord, le gradient thermique diminue $\left(G_{2}=0,010^{\circ} \mathrm{C} / \mathrm{m}\right)$ suite aux importantes circulations d'eau dans la zone de marbres de Veglia (débit linéaire égal à $0,203 \mathrm{l} / \mathrm{s} / \mathrm{m}$ ). L'anomalie de la zone de Teggiolo apparaît également assez nettement sur le diagramme épaisseurs de couverture - températures (figure $4 b$ ).

\section{Galerie Gothard Alptransit}

Une même anomalie thermique a été enregistrée récemment dans la galerie de reconnaissance Alptransit Gothard à Polmengo (Suisse), où les températures augmentent régulièrement dans les gneiss penniques de Leventina et du Lucomagno (SCHNEIDER, 1992) jusqu'au PM 2500 (Point Métrique mesuré 

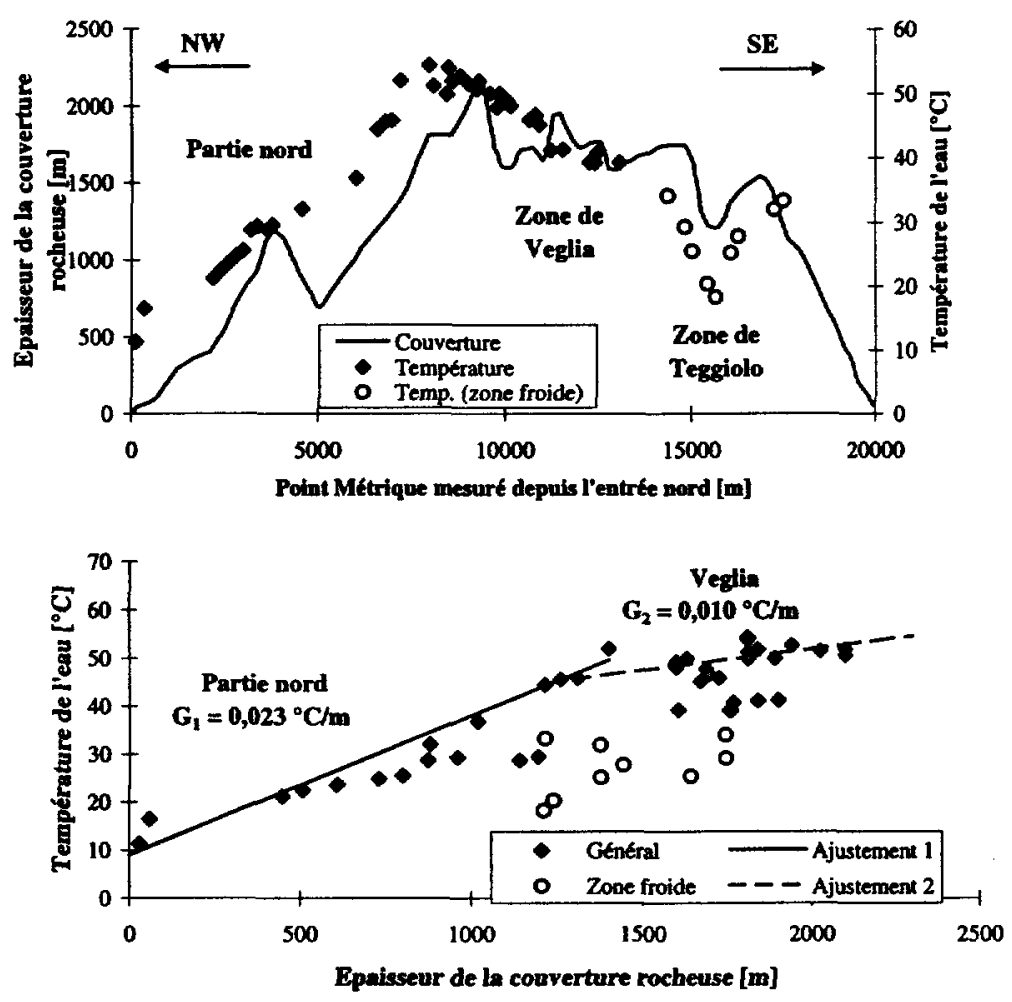

Figure 4 Cas du Simplon.

(a) Profil de la température des venues d'eau et de l'épaisseur de la couverture rocheuse.

(b) Évolution de la température de l'eau en fonction de l'épaisseur de la couverture rocheuse.

Simplon case.

(a) Profiles of water inflows temperatures and rock cover thickness.

(b) Water temperature as a function of rock cover thickness.

depuis l'entrée de la galerie) et se stabilisent aux environs de $27^{\circ} \mathrm{C}$ (SCHNEIDER, 1997) sous une couverture maximale de 1600 mètres (figure 5). Elles subissent, au-delà du PM 4000, le refroidissement causé par la présence de circulations d'eau dans le synclinal métasédimentaire de la Piora Mulde, constitué de dolomies fortement tectonisées et broyées. Le gradient thermique des eaux mesuré dans les formations gneissiques est faible $\left(G=0,013^{\circ} \mathrm{C} / \mathrm{m}\right)$ malgré les petites quantités d'eau qui ont été rencontrées dans ces formations (débit linéaire égal à $0,0015 \mathrm{l} / \mathrm{s} / \mathrm{m}$ ).

Une modélisation mathématique des écoulements et échanges de chaleur dans le massif a permis de faire une prévision des caractéristiques hydrogéologiques et des quantités d'eau circulant dans cette zone (BUSSLINGER et RYBACH, 1997). 

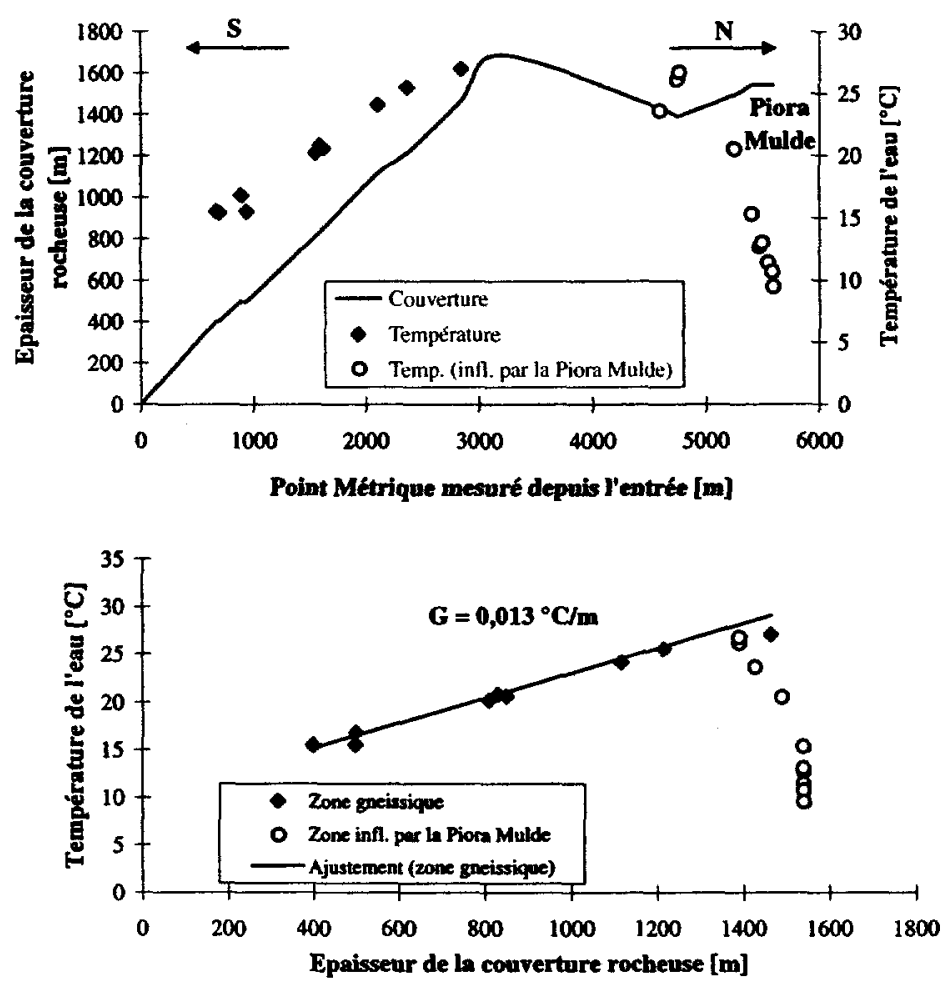

Figure 5 Cas du Gothard AlpTransit.

(a) Profil de la température des venues d'eau et de l'épaisseur de la couverture rocheuse.

(b) Évolution de la température de l'eau en fonction de l'épaisseur de la couverture rocheuse.

\section{Gothard Alptransit case.}

(a) Profiles of water inflows temperatures and rock cover thickness.

(b) Water temperature as a function of rock cover thickness.

\section{3 - SYNTHÈSE}

La revue de ces cinq cas a permis de mettre en évidence diverses anomalies locales d'origine topographique (cas des anomalies positives) et d'origine hydrogéologique (cas des anomalies négatives). Les gradients thermiques des eaux calculés sont résumés dans le tableau 1.

Les gradients thermiques des eaux varient d'un ouvrage à l'autre en fonction de la quantité d'eau drainée par celui-ci. Le débit linéaire et le gradient thermique possèdent une corrélation négative, illustrée sur la figure 6 . Plus il y a d'eau atteignant le tunnel en cours de percement, et plus il y avait donc de 
Tableau 1 Gradients thermiques des eaux calculés dans divers ouvrages souterrains des Alpes. Comparaison avec les débits linéaires.

Table 1 Water thermal gradients calculated in some alpine underground works. Comparison with linear discharge rates.

\begin{tabular}{|c|c|c|c|}
\hline Ouvrage & $\begin{array}{c}\text { Gradient thermique } \\
\text { des eaux }\left[{ }^{\circ} \mathrm{C} / \mathrm{m}\right]\end{array}$ & $\begin{array}{l}\text { Débit linéaire } \\
{[\mathrm{l} / \mathrm{s} / \mathrm{m}]}\end{array}$ & Situation \\
\hline Vereina & $\begin{array}{l}0,018 \\
0,016\end{array}$ & $\begin{array}{l}0,003 \\
0,006\end{array}$ & $\begin{array}{l}\text { Tronçon nord } \\
\text { Tronçon sud }\end{array}$ \\
\hline Gothard N2 & 0,015 & 0,020 & $\begin{array}{l}\text { Monte Prosa } \\
\text { (PM 0-5500-S) }\end{array}$ \\
\hline Mont-Blanc & $\begin{array}{l}0,016 \\
0,007\end{array}$ & $\begin{array}{l}0,008 \\
0,193\end{array}$ & $\begin{array}{l}\text { Schistes cristallins } \\
\text { Granite central }\end{array}$ \\
\hline Simplon & $\begin{array}{l}0,023 \\
0,010\end{array}$ & $\begin{array}{c}0,0004 \\
0,203\end{array}$ & $\begin{array}{l}\text { Gneiss Berisal } \\
\text { Zone de Veglia }\end{array}$ \\
\hline Gothard ATG & 0,013 & 0,0015 & $\begin{array}{l}\text { Gneiss Leventina } \\
\text { (PM } 0-2500)\end{array}$ \\
\hline
\end{tabular}

potentialité d'écoulement d'eau dans le massif avant le percement de l'ouvrage, plus le gradient thermique mesuré est faible. C'est l'effet réfrigérant des circulations d'eau. Les eaux s'infiltrant à haute altitude sont froides (température proche de $0^{\circ} \mathrm{C}$ ) et contribuent à diminuer les températures à l'intérieur du massif. En coordonnées semi-logarithmiques, le meilleur ajustement est une droite dont l'équation est $\ln q=-417^{*} G+1,67$. Quand le débit linéaire devient négligeable (de l'ordre de $10^{-4} \mathrm{l} / \mathrm{s} / \mathrm{m}$ ), le gradient thermique des eaux tend vers une valeur de $0,26^{\circ} \mathrm{C} / \mathrm{m}$, valeur légèrement inférieure, suite au non confinement des massifs étudiés, au gradient géothermique mondial $\left(G=0,030^{\circ} \mathrm{C} / \mathrm{m}\right)$. Sur ce diagramme, le débit linéaire dans la galerie du Gothard Alptransit (ATG) apparaît hors corrélation : des circulations d'eau non rencontrées par l'ouvrage pourraient exister dans le massif.

Pour des raisons relatives aux hypothèses simplificatrices de ce travail (non prise en compte de l'effet du relief notamment), une utilisation prudente de la relation mise en évidence à la figure 6 doit être envisagée. Elle permet par exemple de situer un ouvrage en cours de percement dans la zone des débits élevés du Mont-Blanc et du Simplon (zone de marbres) ou dans la zone des faibles débits du Simplon (gneiss de Berisal) et de la Vereina. Il serait dangereux de se contenter de cette relation pour tenter un pronostic précis des venues d'eau dans le tunnel. 


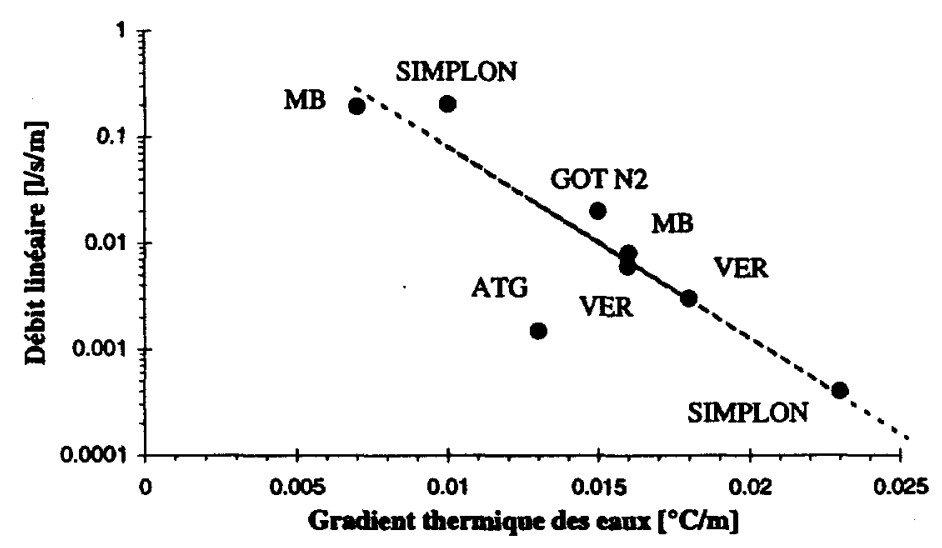

Figure 6 Relation entre le débit linéaire et le gradient thermique des eaux dans divers ouvrages souterrains.

(MB : Mont-Blanc ; GOT-N2 : Gothard-N2 ; VER : VEREINA ; ATG : Gothard Alptransit).

Relation between linear discharge rate and water thermal gradient in some underground works

(MB : Mont-Blanc ; GOT-N2 : Gothard-N2 ; VER : VEREINA ; ATG : Gothard Alptransit).

\section{CONCLUSIONS}

Le suivi des températures des venues d'eau dans cinq ouvrages souterrains profonds dans les Alpes a permis de mettre en évidence l'influence des circulations d'eau et, indirectement, de la perméabilité du massif sur ce paramètre. À l'inverse, il devient dès lors possible, lors du percement d'un ouvrage souterrain, d'améliorer la prévision des venues d'eau à partir des données de températures mesurées en cours d'avancement. Deux types de cas sont possibles : l'observation d'une anomalie locale de température liée à une zone aquifère très localisée ou la baisse du gradient thermique des eaux lié à des venues d'eau diffuses dans le massif.

Des anomalies locales de températures, corrélées ensuite avec de fortes venues d'eau sur de petits tronçons de tunnel ont été mises en évidence dans les tunnels du Simplon, du Mont-Blanc et du Gothard-AT. De telles anomalies de températures apparaissent plusieurs centaines de mètres avant la rencontre de la zone aquifère à l'origine de l'anomalie : la mesure de température constitue donc un outil de prévision des fortes venues d'eau localisées dans une zone aquifère particulière. L'utilisation de modélisations mathématiques tridimensionnelles permet d'améliorer de façon quantitative cette prévision, en tenant compte de la géométrie du problème, de l'hétérogénéité et de l'anisotropie des caractéristiques hydrogéologiques et thermiques des terrains et des conditions aux limites. 
La comparaison des gradients thermiques des eaux à l'échelle du massif avec les débits linéaires drainés par les ouvrages souterrains dans ces massifs permet de déterminer une relation entre ces deux caractéristiques du massif. Cette relation permet d'approcher, à partir du gradient thermique global d'un massif, les quantités d'eau moyennes à anticiper lors de sa traversée par un ouvrage souterrain.

Ces résultats montrent que la mesure de la température de l'eau en cours d'avancement d'un ouvrage souterrain constitue un outil de prévision efficace et peu coûteux des venues d'eau. Dans tous les cas, il convient de tenir compte des anomalies engendrées par le relief ; celles-ci peuvent être importantes dans de tels massifs montagneux. Une modélisation tridimensionnelle du transport de chaleur dans le massif s'avère dans tous les cas nécessaire pour améliorer la précision des prévisions.

\section{REMERCIEMENTS}

Les principaux résultats exposés dans cet article ont été acquis durant un travail de thèse réalisé à l'École Polytechnique Fédérale de Lausanne (thèse EPFL $\left.n^{\circ} 1769,1998\right)$ et en partie financé par la Fondation EVIAN.

\section{RÉFÉRENCES BIBLIOGRAPHIQUES}

BAIR E.S., PARIZEK R.R., 1978. Detection of permeability variations by a shallow geothermal technique. Ground Water, 16(4), 254-263.

BUSSLINGER A., RYBACH L., 1996. Prognosis of rock temperatures and water inflow zones in deep tunneling - examples from the NEAT-project, Swiss Alps. In: "Tunnels for People", GOLSER, HINKEL and SCHUBERT (Ed.), pp. 9-14.

BUSSLINGER A., RYBACH L., 1997. Sondiersystem Piora-Mulde. Geothermische Untersuchungen. 3D-Modellierungen, Interner Bericht Nr 2007. Institut für Geophysik. ETH Zurich. 27 p.

CARTWRIGHT K., 1968. Thermal prospecting for groundwater, Water Resour. Res., $4(2), 395-401$.

DONALDSON J.G., 1962. Temperature gradients in the upper layers of the earth's crust due to convective water flows. J. Geophys. Res., 67, 3449-3459.
GOY L., FABRE D., MENARD G., 1996. Modelling of Rock Temperatures for Deep Alpine Tunnel Projects. Rock Mech. Rock Engng., 29(1), 1-18.

GUDEFIN H., 1967. Observations sur les venues d'eau au cours du percement du tunnel sous le Mont-Blanc. Bull. B.R.G.M. 4, 95-107.

GUICHONET P. et BERNIERI U., 1966. Historique de la percée du Mont-Blanc. La réalisation. Aoste II, $400 \mathrm{p}$.

KELLER F., LOCHER, T., 1994. Die Geologie des Zugwald- und Vereinatunnels. Schweizer Ingenieur und Architekt, 44, 892-896.

KELLER F., WANNER H., SCHNEIDER T.R., 1987. Geologischer Schlussbericht. Gotthard-Strassentunnel. Beiträge zur geologie der Schweiz. Geotechnische serie 70.

KEYS W.S., BROWN R.F., 1978. The use of temperature logs to trace the movement 
of injected water. Ground Water, 16(1), $32-48$.

MARÉCHAL J.C., 1998. Les circulations d'eau dans les massifs cristallins alpins et leurs relations avec les ouvrages souterrains. Th. Doct. Ecole Polytechnique Fédérale de Lausanne, no 1769, $296 \mathrm{p}$.

MARECHAL J.C., PERROCHET P., TACHER L., 1999. Long-term simulations of thermal and hydraulic characteristics in a mountain massif as a tool to predict water inflows in underground works: the Mont-Blanc case study. Hydrogeology Joumal, 7/4, 341-354.

SCHARDT H., 1905. Les eaux souterraines du tunnel du Simplon. La Géographie, bull. Soc. Géograph., 11, 82-96.

RYBACH L., PFISTER M., 1994a. How to predict rock temperatures for deep Alpine tunnels. J. Applied Geophys., 31, 261-270.

RYBACH L. et PFISTER M., 1994b. Temperature predictions and predictive temperatures in deep tunnels. Rock Mech. Rock Engng., 27(2), 77-88.
SCHNEIDER T.R., 1992. Geologie GotthardBasistunnel. Schweizer Ingenieur und Architekt Dokumentation D 085., 15-24.

SCHNEIDER T.R., 1997. Rapports géologiques sur la galerie AlpTransit Gothard. Rapports Inédits.

SMITH L., CHAPMAN D.S., 1983. On the thermal effects of groundwater flow, 1 , Regional scale systems. J. Geophys. Res., 88, 593-608.

STALLMAN R.W., 1963. Computation of groundwater velocity from temperature data. U.S. Geol. Surv. Water Supply Pap., 1554-H, 36-46.

TANIGUCHI M., 1993. Evaluation of Vertical Groundwater Fluxes and Thermal Properties of Aquifers Based on Transient Temperature-Depth Profiles. Water Resour. Res., 29(7), 2021-2026.

WOODBURY A.D., SMITH L., 1985. On the thermal effects of three-dimensional groundwater flow. J. Geophys. Res., 90, 759-767. 